\title{
Lidil
}

Revue de linguistique et de didactique des langues

$62 \mid 2020$

Recherches actuelles en didactique du lexique : avancées, réflexions, méthodes

\section{Introduction. - Recherches actuelles en didactique du lexique : avancées, réflexions, méthodes}

Ophélie Tremblay et Dominic Anctil

\section{(2) OpenEdition}

\section{Journals}

Édition électronique

URL : http://journals.openedition.org/lidil/8322

DOI : $10.4000 /$ lidil.8322

ISSN : 1960-6052

Éditeur

UGA Éditions/Université Grenoble Alpes

Édition imprimée

ISBN : 978-2-37747-226-0

ISSN : $1146-6480$

Référence électronique

Ophélie Tremblay et Dominic Anctil, « Introduction. - Recherches actuelles en didactique du lexique avancées, réflexions, méthodes », Lidil [En ligne], 62 | 2020, mis en ligne le 03 novembre 2020, consulté le 05 novembre 2020. URL : http://journals.openedition.org/lidil/8322 ; DOI : https://doi.org/10.4000/ lidil.8322

Ce document a été généré automatiquement le 5 novembre 2020.

(C) Lidil 


\title{
Introduction. - Recherches actuelles en didactique du lexique : avancées, réflexions, méthodes
}

\author{
Ophélie Tremblay et Dominic Anctil
}

\section{Introduction}

1 La didactique du lexique, comme discipline de recherche et de formation, se développe depuis plusieurs années de façon soutenue, ce dont témoignent diverses publications ${ }^{1}$, numéros de revues ${ }^{2}$ et manifestations scientifiques ${ }^{3}$ qui ont contribué à structurer ce champ de recherche plus activement depuis une bonne quinzaine d'années. Ce numéro s'inscrit d'ailleurs dans le prolongement de colloques et de symposiums en didactique du lexique que nous avons organisés depuis 2015 et qui ont réuni chaque fois plus d'une dizaine de communications et une vingtaine de participantes et de participants. La majorité des contributions qui figurent dans ce numéro présentent ainsi des travaux que nous avons pu suivre de près au fil des dernières années. Nous croyons que la communauté qui s'est construite progressivement à travers ces rencontres a contribué à l'essor de la recherche dans notre domaine, grâce, entre autres, à ces espaces d'échange et de diffusion. Le regard que nous jetons dans ce numéro sur les tendances actuelles en didactique du lexique, à travers les huit articles qui le composent (ainsi que les deux articles de la section Varia), a ainsi pour but de témoigner aussi bien de certains acquis théoriques ou méthodologiques que de pistes didactiques prometteuses, sans oublier les problèmes de recherche ou de formation qui persistent et les questions nouvelles qui se posent. Bien sûr, les travaux présentés ici ne représentent qu'une partie de l'ensemble des recherches menées dans le domaine - il nous a d'ailleurs fallu faire un choix parmi la vingtaine de propositions reçues pour la réalisation de ce numéro. Néanmoins, il nous semble que les contributions retenues apportent chacune à leur manière un éclairage sur différentes questions vives de la discipline. 
2 Diriger ce numéro de Lidil sur la didactique du lexique, en nous plongeant dans les différentes contributions sollicitant différents cadres de référence et postures didactiques, nous a menés à poursuivre certaines réflexions autour de deux notions clés, qui sous-tendent les avancées de la discipline : la conceptualisation même de l'objet lexique et celle de compétence lexicale, explorée notamment à travers sa dimension affective. Les prochaines sections visent à rendre compte de ces réflexions et à faire voir la nécessité de disposer d'un cadrage théorique commun pour soutenir à la fois la conception des dispositifs didactiques (et des ressources lexicales), leur mise en œuvre et l'analyse de leurs effets ou de leurs retombées sur les apprentissages lexicaux des apprenants, notamment dans une perspective évaluative.

\section{Appréhender le lexique comme un réseau pour mieux l'enseigner}

3 Pour la lectrice ou le lecteur ayant déjà fréquenté les écrits en didactique du lexique, la distinction entre les notions de lexique et vocabulaire n'est sans doute plus à faire. Permettons-nous tout de même de la rappeler, au bénéfice de celles et de ceux pour qui ces deux termes seraient perçus comme des synonymes. Le terme vocabulaire désigne l'ensemble des mots maitrisés par un individu (en réception ou en production), ou encore l'ensemble des mots d'un texte; en ce sens, le vocabulaire représente un sousensemble du lexique. Le terme lexique désigne quant à lui l'ensemble des unités lexicales d'une langue, ce qui dépasse la seule notion de « mot». En effet, le lexique est composé de différents types d'« entités lexicales» (Polguère, 2016) : lexèmes (obéir, squelette, irritable), locutions (coup de foudre, soupe au lait, manger les pissenlits par la racine), collocations et cooccurrences (prononcer un discours, facture salée, travailler d'arrachepied), proverbes ou maximes (Tel père, tel fils; Tant va la cruche à l'eau qu'à la fin elle se casse ; Les absents ont toujours tort), pragmatèmes (Défense de stationner, Ouvert $24 \mathrm{~h}$ ). Le lexique se présente donc comme un ensemble organisé d'unités lexicales liées entre elles par des liens paradigmatiques (synonymie, antonymie, hyperonymie, etc.) et syntagmatiques (cooccurrence). De récents travaux défendent en ce sens l'idée que le lexique fonctionnerait comme un véritable réseau (Polguère, 2014; Schur, 2007 ; Wilks \& Meara, 2002), connectant les unités lexicales sur la base de liens divers au sein du lexique mental de l'apprenant (Aitchison, 2012). Cette vision du lexique comme réseau convoque des façons renouvelées d'apprendre et d'enseigner le vocabulaire.

4 En effet, d'un point de vue didactique, la prise en compte du fonctionnement systémique du lexique contribue à la mise en place de démarches d'enseignement qui visent non seulement l'accroissement du vocabulaire en termes quantitatifs, mais aussi en termes qualitatifs (Grossmann, 2011, 2018), par une mise au jour des relations qui structurent le lexique et des phénomènes qui lui sont propres. C'est pour cette raison que nous préférons parler d'enseignement du lexique plutôt que d'enseignement du vocabulaire : enseigner efficacement le lexique, c'est permettre aux élèves d'élargir leur vocabulaire, bien sûr, mais aussi d'accroitre leur compréhension du système lexical.

5 Nous savons par ailleurs que les unités lexicales, au-delà de leur forme et de leur sens, possèdent différentes propriétés qui régissent leur usage en situation de discours (catégorie grammaticale, régime, appartenance à un registre de langue, genre nominal, etc.), ce qui appelle une prise en compte des interactions entre lexique et syntaxe aussi 
bien dans les dispositifs d'enseignement que dans les analyses de textes d'élèves (Grossmann, 2018). Afin de développer leur vocabulaire, de même que leur compétence à lire et à orthographier, les apprenants doivent donc se familiariser avec ces différentes dimensions de la connaissance lexicale (Beck, McKneow \& Kucan, 2013; Nation, 2001; Polguère, 2016). La didactique du lexique est donc appelée à repenser l'enseignement du vocabulaire à travers des activités novatrices qui tiennent compte des différentes facettes de la connaissance lexicale, par exemple en délaissant les approches traditionnelles prenant appui sur des listes de "mots" présentés hors contexte pour privilégier plutôt le recours à de nouvelles ressources didactiques qui mettent mieux en évidence le caractère "connecté" du lexique (dictionnaires électroniques, corpus, etc.). Comme le fait remarquer une des enseignantes de l'étude que Marie-Noëlle Roubaud présente dans ce numéro : «Comprendre que le vocabulaire est organisé, c'est plus important qu'apprendre des listes de mots!»

\section{Caractériser la compétence lexicale pour mieux en soutenir le développement et l'évaluation}

6 La conception du lexique comme un immense réseau structurant des milliers d'entités lexicales, dont la connaissance est multidimensionnelle, nous invite à réfléchir à la notion même de compétence lexicale, au cœur de nos préoccupations comme didacticiennes et didacticiens du lexique.

7 Grâce à une recension systématique d'écrits publiés tant en anglais (p. ex. Chapelle, 1998 ; Henriksen, 1999 ; Kieffer \& Lesaux, 2012 ; Marconi, 1997 ; Meara, 1996 ; Nation, 2001; Zareva, Schwanenflugel \& Nikolova, 2005) qu'en français (Corbin, 1980; Grossmann, 2005 ; Nisubire, 2003 ; Simard, 1994 ; Tréville, 2000) et en nous appuyant sur la caractérisation de ce qu'est une compétence (Paquette, 2002), nous avons proposé une définition de la compétence lexicale qui permet de caractériser celle-ci à travers trois composantes: connaissances, habiletés, attitudes (Tremblay, Anctil \& Perron, 2016). Nous détaillons dans les deux tableaux suivants, les éléments « cognitifs » liés aux deux premières composantes, les connaissances et les habiletés. 
Tableau 1. - Caractérisation des connaissances constitutives de la compétence lexicale.

\begin{tabular}{|c|c|c|c|}
\hline \multicolumn{4}{|c|}{$\begin{array}{l}\text { ASPECT COGNITIF - Connaissance des entités lexicales } \\
\text { (lexèmes, locutions, vocables, proverbes, pragmatèmes) }\end{array}$} \\
\hline $\begin{array}{c}\text { Aspect } \\
\text { quantitatif }\end{array}$ & \multicolumn{3}{|c|}{ Aspect qualitatif } \\
\hline $\begin{array}{l}\text { - Nombre de } \\
\text { «mots » } \\
\text { possédés par } \\
\text { un locuteur } \\
\text { (étendue du } \\
\text { vocabulaire). }\end{array}$ & $\begin{array}{l}\text { Forme } \\
\text { - Connaissance des } \\
\text { formes orale et } \\
\text { écrite. } \\
\text { - Connaissance de la } \\
\text { forme } \\
\text { orthographique et } \\
\text { de la structure } \\
\text { phonologique d'un } \\
\text { mot. } \\
\text { - Connaissance } \\
\text { phonétique, } \\
\text { orthographique, } \\
\text { phonologique, } \\
\text { morphologique. }\end{array}$ & $\begin{array}{l}\text { Sens } \\
\text { - Connaissance du } \\
\text { sens. } \\
\text { - Connaissance de la } \\
\text { polysémie d'un } \\
\text { vocable (son sens } \\
\text { propre et ses sens } \\
\text { figurés). } \\
\text { - Connaissance } \\
\text { référentielle. }\end{array}$ & $\begin{array}{l}\text { Combinatoire } \\
\text { - Connaissance du } \\
\text { registre et des } \\
\text { contextes } \\
\text { d'utilisation. } \\
\text { - Connaissance des } \\
\text { propriétés } \\
\text { stylistiques. } \\
\text { - Connaissance des } \\
\text { cooccurrents d'une } \\
\text { unité lexicale. } \\
\text { - Connaissance des } \\
\text { connotations } \\
\text { affectives, } \\
\text { stylistiques, } \\
\text { culturelles associées } \\
\text { à une unité lexicale. }\end{array}$ \\
\hline \multicolumn{4}{|c|}{ ASPECT COGNITIF - Connaissances implicites du système lexical } \\
\hline \multicolumn{4}{|c|}{$\begin{array}{l}\text { - Organisation et propriété du lexique. } \\
\text { - Procédés de dérivation morphologique. } \\
\text { - Polysémie. } \\
\text { - Relations de sens (antonymes, synonymes, hyponymes, hyperonymes). } \\
\text { - Réseaux d'associations qui lient les mots aux autres mots. } \\
\text { - Relations syntagmatiques. }\end{array}$} \\
\hline
\end{tabular}

Tableau 2. - Caractérisation des habiletés constitutives de la compétence lexicale.

\begin{tabular}{|c|c|c|}
\hline \multicolumn{3}{|c|}{ ASPECT COGNITIF - HABILETÉS } \\
\hline $\begin{array}{l}\text { Oral } \\
\text { Forme } \\
\text { - Être capable de } \\
\text { prononcer } \\
\text { correctement le mot. } \\
\text { - Être capable de } \\
\text { reconnaitre la forme } \\
\text { orale d'un mot. } \\
\text { - Produire de nouvelles } \\
\text { unités lexicales à partir } \\
\text { de moyens } \\
\text { morphologiques. } \\
\text { Sens } \\
\text { - Produire des } \\
\text { paraphrases. } \\
\text { - Effectuer une } \\
\text { inférence sémantique. } \\
\text { - Être capable d'utiliser } \\
\text { des synonymes et des } \\
\text { antonymes de ce mot. } \\
\text { - Choisir le mot adéquat } \\
\text { pour exprimer un sens } \\
\text { précis. } \\
\text { Combinatoire } \\
\text { - Être capable d'utiliser } \\
\text { un mot selon le degré } \\
\text { de formalité d'une } \\
\text { situation. }\end{array}$ & $\begin{array}{l}\text { Écrit } \\
\text { Forme } \\
\text { - Encoder des sons et } \\
\text { l'orthographe d'un mot. } \\
\text { Sens } \\
\text { - Produire des } \\
\text { paraphrases. } \\
\text { - Choisir le mot adéquat } \\
\text { pour exprimer un sens } \\
\text { précis. } \\
\text { - Être capable d'utiliser } \\
\text { des synonymes et des } \\
\text { antonymes de ce mot. } \\
\text { Combinatoire } \\
\text { - Être capable d'utiliser } \\
\text { des mots qui } \\
\text { apparaissent souvent } \\
\text { avec ce même mot. } \\
\text { - Être capable d'utiliser } \\
\text { ou non un mot selon le } \\
\text { degré de formalité d'une } \\
\text { situation. }\end{array}$ & $\begin{array}{l}\quad \text { Lecture } \\
\text { Forme } \\
\text { - Être capable de } \\
\text { reconnaitre la forme } \\
\text { écrite d'un mot. } \\
\text { Sens } \\
\text { - Effectuer une inférence } \\
\text { sémantique à partir } \\
\text { d'indices contextuels et } \\
\text { morphologiques. } \\
\text { - Être capable de réviser } \\
\text { une interprétation } \\
\text { lexicale en fonction du } \\
\text { contexte. } \\
\text { - Utiliser les indices } \\
\text { lexicaux du texte. } \\
\text { Combinatoire } \\
\text { - Utiliser les règles de } \\
\text { construction de la langue } \\
\text { pour interpréter le sens } \\
\text { des lexèmes et des } \\
\text { collocations. }\end{array}$ \\
\hline
\end{tabular}


Ce qu'illustrent ces deux tableaux, c'est la diversité des éléments de contenu pouvant être abordés à travers l'enseignement du lexique, qu'il s'agisse de connaissances ou d'habiletés.

Une troisième composante de la compétence lexicale touche quant à elle à la dimension des attitudes qui interviennent dans le développement conjoint des connaissances et des habiletés liées à la maitrise des mots. Il s'agit d'une dimension qui attire notre attention depuis un certain temps (Lavoie, Bélanger \& Tremblay, 2017; Tremblay \& Anctil, 2015 ; Tremblay \& Gagné, 2020), dans la foulée de travaux anglo-saxons sur le concept de Word Consciousness (Graves \& Watts-Taffe, 2008 ; Scott, Skobel \& Wells, 2008). Ces travaux ont permis de dégager des exemples de manifestations de cette attitude constitutive de la compétence lexicale, attitude que nous avons désignée plus spécifiquement sous le nom de «sensibilité lexicale» (Tremblay \& Gagné, 2020 ; Tremblay, à paraitre) :

- faire preuve d'enthousiasme face à l'apprentissage de nouveaux mots ou de nouvelles expressions ;

- être sensible aux contextes d'utilisation de nouveaux mots ;

- être motivé à apprendre des mots nouveaux ;

- remarquer des mots nouveaux utilisés par les autres et leur demander ce que ces mots veulent dire;

- aimer partager ses découvertes lexicales avec son entourage ;

- manifester un intérêt à apprendre la signification d'un mot nouveau rencontré dans un texte et à en comprendre les subtilités ;

- aimer ouvrir le dictionnaire pour y chercher de l'information ;

- aimer jouer avec les mots (leur sens, leur forme orale ou écrite, leur usage);

- accorder de l'importance au choix et à la précision des mots à l'écrit ou à l'oral ;

- accorder de l'importance au fait d'adapter son vocabulaire en fonction des situations de communication.

Comme en témoignent plusieurs des contributions de ce numéro, une attitude curieuse et enthousiaste face aux mots et à leur apprentissage semble jouer un rôle dans le développement $d u$ vocabulaire et la maitrise du sens et de l'usage des mots. Un enseignement lexical efficace doit donc soutenir le développement de cette composante affective de la compétence lexicale.

11 En somme, la caractérisation proposée pour chacune des composantes de la compétence lexicale montre la diversité des connaissances, des habiletés et des attitudes qu'il est possible de développer à travers l'enseignement du lexique, quel que soit le degré scolaire considéré. Chacune des composantes et de ses éléments constitutifs apparait ainsi comme un possible objet d'enseignement et d'apprentissage ou d'évaluation.

Par ailleurs, des connaissances d'ordre «méta " sur les différentes dimensions impliquées dans la connaissance des unités lexicales et sur les liens structurant le lexique peuvent contribuer à développer la connaissance lexicale de façon plus systématique, organisée et efficace. Par exemple, si l'habileté à utiliser un synonyme dans un contexte de communication à l'oral ou à l'écrit relève de la compétence lexicale $\mathrm{du}$ locuteur, la capacité à décrire explicitement la relation unissant deux unités lexicales (synonymie, antonymie, hyperonymie, copolysémie, etc.) relève, elle, d'une habileté qu'on qualifiera de métalexicale. La compétence métalexicale correspondrait ainsi à un ensemble de connaissances, d'habiletés et d'attitudes qui permettraient de 
réfléchir sur le lexique, de décrire plus formellement les unités lexicales et leurs relations tout en manifestant une attitude de curiosité ou d'enthousiasme face à l'usage des notions descriptives (et non pas des "mots » eux-mêmes). Il s'agit là d'une compétence qu'ont développée les lexicographes, par exemple, mais qui est aussi à la portée des enseignantes et des enseignants et de leurs élèves! Des travaux en didactique du lexique se sont d'ailleurs intéressés à la question des notions de base à maitriser pour l'enseignement du lexique (Anctil, 2011; Grossmann, 2011; Tremblay, 2009) et à leur progression dans un dispositif de formation universitaire en enseignement (Tremblay \& Polguère, 2014). Plusieurs contributions de ce numéro (p. ex., Aurnague \& Garcia-Debanc, Lux Pogodalla, Roubaud, Vénérin-Guénez) mettent également de l'avant l'importance, pour l'enseignant.e, de maitriser ces notions afin d'accompagner efficacement les élèves dans leurs réflexions et leurs apprentissages sur le lexique et son fonctionnement. Cela dit, la question des interactions entre compétence lexicale et compétence métalexicale reste à approfondir, dans la foulée de la proposition de caractérisation de la notion de compétence lexicale que ce texte de présentation nous a permis de poser et des apports de chacune des contributions du numéro.

\section{Présentation des contributions}

13 Ce numéro dresse un portrait des recherches actuelles qui contribuent à l'évolution de la didactique du lexique autour de trois axes : 1) les pratiques d'enseignement, 2) les ressources lexicales et 3) l'évaluation de la compétence lexicale. Les contributions font état de recherches menées dans différents coins du monde (France, île de La Réunion, Québec), auprès de publics scolaires variés (maternelle, primaire, université ; contexte plurilingue ; milieu défavorisé). La section Varia présente également un entretien avec Francis Grossmann qui permet de retracer quelques éléments clés du parcours de ce chercheur, éclairant au passage sa contribution à la didactique du lexique comme discipline de recherche.

\subsection{Des pratiques innovantes pour l'enseignement du lexique}

Cinq contributions du numéro témoignent de démarches d'enseignement prometteuses expérimentées dans les classes du préscolaire et du primaire et illustrent l'efficacité de dispositifs qui mobilisent la réflexion des élèves sur les mots, tout en leur permettant de développer leur maitrise tant du sens que des usages des unités lexicales.

L'article de Marie-Noëlle Roubaud présente d'abord le dispositif du mot-vedette, à travers le compte rendu d'une recherche menée dans 35 classes de maternelle au fil de quatre années (de 2014 à 2018). Dans ce dispositif, c'est précisément une entrée par le mot qui structure les discussions et la "collection" de mots proposées dans la démarche, qui se déroule sur plusieurs jours. Pour commencer, l'enseignante fait émerger les représentations des élèves à propos du mot-vedette choisi. Ces réponses sont notées par l'enseignante en vue d'une catégorisation, le deuxième jour, à partir de questions précises permettant de formuler une définition du mot-vedette et de rechercher des mots thématiquement ou syntaxiquement liés à ce dernier. Ces questions visent à étudier les facettes de la connaissance d'un mot, ainsi que son ou ses référents potentiels. Le dernier jour, les élèves, à l'aide de l'enseignante, créent une 
affiche consacrée au mot-vedette, sur laquelle figurent deux phrases (une produite par un élève et l'autre tirée d'un album jeunesse). Le compte rendu d'expérimentation présenté dans l'article met en valeur le rôle catalyseur de la démarche du mot-vedette pour développer la compétence lexicale des élèves, en particulier en ce qui a trait à la manifestation d'une attitude de curiosité envers les mots (et ce, tant chez les élèves que chez les enseignantes). On constate ainsi que même à un très jeune âge, les enfants sont capables d'une activité réflexive, qu'ils sont à même d'organiser les mots, de s'initier aux relations lexicales en plus de développer leur habileté à produire des phrases à partir des mots choisis. Les enseignantes rapportent quant à elles avoir porté davantage attention à l'intérêt personnel de chaque élève envers les mots, ce qui dénote une prise en compte de la composante affective de la compétence lexicale.

Le même type d'activité réflexive autour des mots se rencontre également dans la recherche de Christine Vénérin-Guénez, qui présente une étude menée dans six classes réunionnaises de $\mathrm{CM} 2,6^{e}$ et $5^{e}$ (élèves âgés de 11 à 13 ans), entre février et mai 2018. Le dispositif expérimenté visait à mesurer l'influence de plusieurs séances lexicales orales sur le réinvestissement en production écrite. Ce dispositif fait appel à des rappels de récits de contes (exploitant le champ sémantique lié au thème de la ruse), ainsi qu'à des entretiens métalexicaux, en vue d'un travail d'écriture d'invention. Les données analysées se fondent sur 463 productions écrites et huit heures de vidéos enregistrées dans quatre classes. Dans l'ensemble, le réinvestissement s'observe dans la variété des reformulations et des unités lexicales choisies pour les reprises (verbes simples, noms, adjectifs ou groupes adjectivaux), en particulier dans le troisième rappel écrit. Ce qui ressort ainsi de l'étude réalisée, c'est l'intérêt de la démarche des entretiens d'explicitation lexicale menés dans les classes à partir de choix lexicaux repérés par la chercheuse dans les productions des élèves. Ces entretiens permettent de mieux comprendre les choix des élèves et de les aider ensuite à mieux utiliser les mots à l'oral et à l'écrit, en développant leur conscience réflexive. À cet égard, la chercheuse souligne l'émergence de la curiosité et de l'engagement des élèves, en particulier d'élèves faibles scripteurs ou faibles parleurs, ce qui encore une fois met en relief la sensibilité lexicale dans le développement de la compétence lexicale.

Faisant écho aux deux articles précédents, Constance Lavoie, Patricia-Anne Blanchet et Martine Pellerin proposent elles aussi d'approcher le travail lexical sur les relations sémantiques à travers une approche discursive. La Communauté de recherche lexicale qu'elles proposent s'inspire des communautés de recherche philosophique utilisées en philosophie pour enfants et vise le développement de stratégies lexicales et métacognitives pour apprendre à repérer et à analyser les relations qui existent entre les mots (famille morphologique, synonymie, antonymie). L'article s'intéresse principalement à la première étape de la démarche, une discussion lexicale autour d'un mot connu des élèves. Lors de cette étape, les élèves prennent tantôt part à la discussion ou adoptent une position d'observateurs actifs ayant pour tâche de relever les mots en lien avec le mot discuté. Par l'analyse détaillée de l'enregistrement d'une discussion autour du verbe rechercher menée par 19 élèves de 8-9 ans de milieu plurilingue et défavorisé, et des cartes de relevés de mots produites par ces élèves, les auteures font ressortir toute la richesse de ce type d'approche pour créer un corpus de mots pouvant servir de tremplin pour l'exploration des relations lexicales lors des étapes subséquentes de la communauté de recherche lexicale. 
18 La contribution de Michel Aurnague et Claudine Garcia-Debanc fait écho à notre réflexion sur la compétence lexicale des enseignantes et des enseignants en s'intéressant à l'impact de leurs conceptions de la structure du lexique sur leurs interventions didactiques dans le cadre d'une ingénierie didactique portant sur les verbes de déplacement. En revisitant les données d'une recherche collaborative menée dans des classes de cycle 3 (élèves de 8 à 11 ans), analysées par ailleurs sous un angle linguistique dans de précédents articles (Aurnague \& Garcia-Debanc, 2016a, 2016b), les auteurs s'intéressent ici aux visions différentes de l'organisation du lexique des enseignantes et des enseignants et à leur influence sur les classifications des verbes élaborées par les élèves dans le cadre d'activités lexicales en classe. Par la mise en relation d'extraits filmés lors de ces activités et des regroupements proposés par les élèves des différentes classes (nombre de catégories créées, propriétés sémantiques mobilisées), les auteurs montrent que les interventions des enseignantes et des enseignants sont guidées par leur façon d'appréhender le système lexical. Ce constat mène à une intéressante réflexion sur les connaissances et compétences lexicales et métalexicales requises, chez les enseignantes et les enseignants, pour la mise en œuvre d'un enseignement rénové du lexique.

19 Rachel Berthiaume, Dominic Anctil et Daniel Daigle présentent quant à eux une étude quasi expérimentale visant à comparer une approche traditionnelle de l'enseignement du vocabulaire (le recours à des listes orthographiques de mots à mémoriser) à une approche de l'enseignement de mots ciblés plus en phase avec les recommandations issues de la recherche. Cette approche consiste à mener un enseignement explicite des mots à travers des activités visant les différentes dimensions de la connaissance lexicale (sens, forme, utilisation). Douze classes ordinaires de $4^{\mathrm{e}}$ année primaire (élèves de 9 à 10 ans) ont participé à l'étude, qui mesurait l'apprentissage de 72 mots sur une période de dix semaines: deux groupes n'ont reçu aucun enseignement, deux ont procédé à l'aide de listes de mots et les six groupes expérimentaux ont reçu un enseignement explicite et pluridimensionnel selon différentes modalités (activités à l'oral seulement, ou activités faisant appel à l'oral et à l'écrit). Les résultats démontrent que les élèves ayant bénéficié d'un enseignement pluridimensionnel ont progressé de façon significative par rapport aux élèves ayant reçu un enseignement à partir de listes de mots, tant du point de vue de la connaissance des mots que de l'orthographe. Ces résultats prometteurs nous invitent à repenser la façon d'approcher l'enseignement de mots ciblés et à remettre en question la place centrale qu'occupe l'orthographe lexicale en classe.

\subsection{Des ressources numériques pour l'enseignement du lexique}

20 Outre les dictionnaires électroniques, de nombreuses ressources numériques sont susceptibles de soutenir l'enseignement du lexique. C'est par exemple le cas du dictionnaire de synonymes $\mathrm{Crisco}^{4}$, du site du Centre national de ressources textuelles et lexicales $^{5}$, des concordanciers ${ }^{6}$ ou encore des moteurs de recherche permettant la consultation de corpus de phrases liées à des listes de mots issus de manuels scolaires ${ }^{7}$ ou d'œuvres de littérature jeunesse québécoise ${ }^{8}$. Si ces ressources ne sont pas toutes destinées en priorité aux enseignant.e.s ou aux apprenant.e.s, elles présentent néanmoins un potentiel didactique prometteur, notamment parce qu'elles facilitent 
l'accès aux informations sur les mots. Dans ce numéro, deux ressources numériques innovantes sont présentées.

La première est le Réseau lexical du français (Lux-Pogodalla \& Polguère, 2011 ; Polguère \& Sikora, 2013), dont Veronika Lux-Pogodalla présente une utilisation pour réfléchir, de façon exploratoire, à la résolution de problèmes de confusions entre le sens d'unités lexicales de sens courant et d'unités lexicales de sens spécialisé en mathématiques. À travers un parcours minutieux dans les représentations et définitions proposées dans le Réseau lexical du français pour les unités lexicales examinées, elle met en évidence l'ébauche d'un patron d'erreur potentiel (ou "motif ») entre sens courants et sens disciplinaires. La chercheuse constate ainsi que la confusion concerne systématiquement deux unités d'un vocable polysémique (deux copolysèmes), que l'élève n'a pas correctement identifiées comme distinctes. L'exploration du réseau permet d'illustrer l'intérêt de la représentation graphique pour soutenir la compréhension des différents sens examinés et des relations lexicales spécifiques qu'entretient chacune des unités lexicales. La chercheuse fait d'ailleurs l'hypothèse que de telles visualisations pourraient soutenir les enseignantes et les enseignants dans leur compréhension des liens entre les unités lexicales en jeu dans les confusions rencontrées par les élèves, de même que dans les explications à fournir à ces derniers pour les aider à mieux comprendre et à utiliser les unités lexicales, en particulier en contexte disciplinaire.

La seconde ressource faisant l'objet d'une contribution dans ce numéro est la base de données lexicales ReSyf, présentée par Núria Gala et Ludivine Javourey-Drevet. Cet outil accessible en ligne permet de naviguer à travers des réseaux d'unités lexicales désambigüisées pour découvrir une riche banque de synonymes associés aux différentes acceptions d'un vocable. Il est d'autant plus novateur qu'il s'intéresse à la difficulté des synonymes proposés, en termes de décodage et de compréhension, déterminée sur la base de critères variés (fréquence, longueur, structure syllabique et morphémique, régularité des correspondances graphèmes-phonèmes, etc.). Les auteures présentent d'abord la méthodologie qui a permis de concevoir ReSyf en tirant profit d'une ressource existante (la plateforme participative JeuxDeMots), d'algorithmes de désambigüisation et de modèles statistiques. La ressource lexicale laisse présager d'intéressantes applications didactiques, explorées dans la seconde moitié de l'article, notamment dans une perspective orthopédagogique à travers une expérience de simplification de textes proposés à des élèves en difficulté. Les auteures soulèvent en conclusion le rôle central des connaissances métalexicales dans l'utilisation éclairée de ressources lexicales.

\subsection{L'évaluation de la compétence lexicale}

Que ce soit pour vérifier l'efficacité d'une intervention, pour effectuer un suivi des apprentissages ou encore pour cibler les besoins lexicaux des élèves, l'évaluation des connaissances lexicales est nécessaire. Elle demeure néanmoins une question complexe. À travers la proposition de modélisation de la compétence lexicale présentée plus tôt, il est possible de cibler aussi bien les contenus d'enseignement que les connaissances, habiletés ou même attitudes à évaluer chez les apprenantes et les apprenants. Deux contributions de ce numéro s'intéressent à la question de l'évaluation.

La contribution de Françoise Boch et Julie Sorba aborde spécifiquement cette question et permet d'envisager les types de tâches à proposer pour mesurer la compétence 
lexicale. L'étude exploratoire a comme objectif de déterminer les caractéristiques des bons distracteurs à l'intérieur d'un test QCM évaluant la compétence lexicale. Pour y arriver, les chercheuses ont fait l'analyse des résultats obtenus par des étudiants universitaires à deux exercices, l'un de synonymie, l'autre de reformulation, en examinant les distracteurs choisis au fil de ces exercices. Les observations des chercheuses les conduisent à conclure que les distracteurs viennent influencer les choix des candidats de différentes manières. D'abord, elles notent que leurs résultats confirment, à l'instar d'autres travaux du domaine, que les distracteurs liés sémantiquement à l'unité lexicale testée sont efficaces (synonymes, antonymes ou emplois similaires entre la lexie cible et le distracteur). D'autre part, les distracteurs non reliés sémantiquement paraissent aussi efficaces : c'est le cas de mots partageant une similarité de forme (relation de paronymie). Elles notent cependant que pour confirmer les hypothèses formulées pour expliquer le choix des distracteurs, des recherches permettant la tenue d'entretiens avec les individus testés permettraient de mieux comprendre la source des erreurs.

La contribution de Christine Da Silva-Genest et ses collègues, présentée dans la section Varia du numéro, aborde aussi la question de l'évaluation des apprentissages lexicaux, cette fois dans une perspective orthopédagogique. Dans leur étude exploratoire, les auteurs interrogent la pertinence des items lexicaux présents dans les tests utilisés par les spécialistes pour évaluer le langage des patients et poser des diagnostics. Leur démarche consiste à dresser un inventaire des items lexicaux utilisés dans différents tests de langage pour des enfants de 4 à 8 ans, puis à comparer cet inventaire à une liste de fréquence des formes produites et entendues par les enfants lors d'interactions dans des situations de la vie courante. Leur analyse fait ressortir certaines disparités entre le vocabulaire sollicité réellement en situations authentiques et celui ciblé dans les tests, ce qui remet en question les méthodes de sélection des items lexicaux et d'établissement des scores obtenus par les patients. Les auteurs interrogent aussi la place centrale qu'occupent les tâches de dénomination dans les tests et l'absence de prise en compte du langage spontané.

\section{Conclusion}

Les dispositifs présentés dans les recherches rapportées au fil de ce numéro montrent qu'il existe de multiples points d'entrée pour l'étude du lexique, bien au-delà de l'usage traditionnel des listes de mots. Nous remarquons d'ailleurs que dans la majorité des travaux présentés, l'enseignement se fait dans une perspective intégrée, où le travail sur le lexique s'articule à des démarches qui mobilisent l'écrit (lecture et écriture) et l'oral. Les dispositifs présentés montrent en outre qu'il est possible de dépasser les apprentissages incidents au hasard de la rencontre de nouveaux mots en privilégiant un enseignement "explicite, systématique et programmé », pour reprendre les mots d'Aurnague et de Garcia-Debanc. Nous notons à cet égard une progression des contenus travaillés autour des trois dimensions de la compétence lexicale, qu'il s'agisse de la connaissance lexicale (forme, sens et usage) des habiletés lexicale (reformulation, réflexion sur le sens des mots et leurs relations) ou des attitudes à développer pour mieux apprendre les mots. D'ailleurs, parmi les travaux à entreprendre dans le prolongement des recherches présentées, la question des attitudes nous parait un facteur à observer et à mesurer plus spécifiquement dans les études futures, afin de 
mieux cerner son rôle dans la réussite des dispositifs pour l'accroissement du vocabulaire ou le développement plus global de la compétence lexicale des apprenants.

Sur la question spécifique des "mots de vocabulaire à enseigner ", le présent numéro apporte certaines réponses, qui suggèrent des pistes de réinvestissement en classe pour renouveler les usages traditionnels de la liste de mots : partir d'un mot-vedette, tout en laissant une part de libre choix aux enfants quant aux mots à «explorer» (Roubaud) ; constituer des corpus thématiques de mots caractérisés par des relations sémantiques spécifiques, par exemple les verbes de déplacement (Aurnague \& Garcia-Debanc); choisir des mots liés à une thématique ou un champ lexical, celui de la ruse, par exemple, pour se rappeler oralement un récit puis en écrire un (Vénérin); faire appel à la fréquence et à certaines difficultés orthographiques pour choisir les mots à enseigner (Berthiaume, Anctil \& Daigle ; Gala \& Javourey) ; identifier les mots dont la polysémie peut conduire à des erreurs de compréhension ou d'usage pour les travailler avec les enseignants et les élèves, en particulier dans une perspective interdisciplinaire (Lux-Pogodalla) ou encore, auprès d'apprenants adultes, utiliser des critères tels que les collocations qui accompagnent tel ou tel mot courant afin de choisir quels mots enseigner, mais aussi quelles connaissances lexicales évaluer (Boch \& Sorba). Souhaitons que ces dispositifs trouvent leur place dans les classes tant au primaire qu'au secondaire, qu'il s'agisse du dispositif du mot-vedette, des échanges oraux autour d'unités lexicales en vue d'un réinvestissement à l'écrit, de discussions lexicales à visée philosophique ou encore de l'étude approfondie d'un corpus de verbes thématiquement liés en vue d'en maitriser le sens et les usages.

Nous terminons avec un dernier point commun à la plupart des articles, soit le rôle incontournable de l'enseignant.e dans le développement lexical des élèves, tant comme modèle linguistique manifestant sa sensibilité lexicale que comme professionnel.le de la langue maitrisant des connaissances sur le lexique et son fonctionnement. En ce sens, les contributions nous rappellent la nécessité de revoir la formation initiale et continue des enseignantes et des enseignants, en y intégrant l'apprentissage de notions lexicales et didactiques nécessaires pour la mise en œuvre de pratiques efficaces d'enseignement du vocabulaire.

C'est avec beaucoup de plaisir que nous avons coordonné ce numéro de Lidil sur la didactique du lexique et constaté la vitalité de notre discipline. Évidemment, les développements actuels ouvrent de nouveaux chantiers à explorer et nous souhaitons avoir l'occasion d'y contribuer avec vous au cours des prochaines années.

\section{BIBLIOGRAPHIE}

Aitchison, Jean. (2012). Words in the Mind: An Introduction to the Mental Lexicon ( $4^{\mathrm{e}}$ éd.). Oxford, UK : Wiley-Blackwell.

ANCTIL, Dominic. (2011). L'erreur lexicale au secondaire : analyse d'erreurs lexicales d'élèves de $3^{e}$ secondaire et description du rapport à l'erreur lexicale d'enseignants de français (Thèse de doctorat, 
Université de Montréal, Québec). Disponible en ligne sur <https://papyrus.bib.umontreal.ca/ xmlui/handle/1866/5077>.

AuRnAGUE, Michel \& GARCIA-DEBAnc, Claudine. (2016a). Enseignement du lexique à l'école primaire et modélisations linguistiques : exemples d'activités portant sur des verbes de déplacement strict. Pratiques, 169-170. <https://doi.org/10.4000/pratiques.2990>.

AuRnAGUe, Michel \& GARCIA-DEBAnc, Claudine. (2016b). Les verbes de déplacement comme contenu d'enseignement du lexique à l'école primaire : modélisation linguistique et analyse de productions d'élèves. Dans F. Neveu, G. Bergounioux, M. H. Côté, J. M. Fournier, L. Hriba \& S. Prévost (dir.), Actes du $5^{e}$ Congrès mondial de linguistique française, CMLF 2016. Paris : ILF \& EDP Sciences. <https://doi.org/10.1051/shsconf/20162707001>.

BECK, Isabel L., McKeown, Margaret G. \& KuCAN, Linda. (2013). Bringing Words to Life. Robust Vocabulary Instruction ( $2^{\mathrm{e}}$ éd.). New York : The Guilford Press.

CALAQUE, Élizabeth \& DAVID, Jacques (dir.). (2004). Didactique du lexique : contextes, démarches, supports. Bruxelles : De Boeck

CHAPELLE, Carol. (1998). Construct Definition and Validity Inquiry in SLA Research. Dans L. F. Bachman \& A. D. Cohen (dir.), Second Language Acquisition and Language Testing Interfaces (p. 32-70). Cambridge, UK : Cambridge University Press.

CORBIN, Danielle. (1980). Compétence lexicale et compétence syntaxique. Modèles linguistiques, II(2), 52-138.

Garcia-Debanc, Claudine, Masseron, Caroline \& Ronveaux, Christophe (dir.). (2013). Enseigner le lexique. Namur : Presses universitaires de Namur.

GRAVES, Michael F. \& WATTS-TAFFE, Susan. (2008). For the Love of Words: Fostering Word Consciousness in Young Readers. The Reading Teacher, 62(3), 185-193.

GrossmanN, Francis. (2005). Le rôle de la compétence lexicale dans le processus de lecture et l'interprétation des textes. Dans J.-P. Bronckart, E. Bulea \& M. Pouliot (dir.), Repenser l'enseignement des langues : comment identifier et exploiter les compétences? (p. 161-191). Villeneuved'Ascq : Presses universitaires du Septentrion.

GrossmanN, Francis. (2011). Didactique du lexique : état des lieux et nouvelles orientations. Pratiques, 149-150, 163-183.

GROSSMANN, Francis. (2018). Comment intégrer l'enseignement du lexique dans la production écrite? Disponible en ligne sur <http://www.cnesco.fr/wp-content/uploads/2018/04/

CCEcrits_note_Grossmann.pdf> (consulté le 14 juillet 2019).

Grossmann, Francis, Paveau, Anne-Marie \& Petit, Gérard (dir.). (2005). Didactique du lexique : langue, cognition, discours. Grenoble : ELLUG.

GRosSmanN, Francis \& Plane, Sylvie (dir.). (2008). Les apprentissages lexicaux : lexique et production verbale. Villeneuve-d'Ascq : Presses universitaires du Septentrion.

HENRIKSEN, Birgit. (1999). Three Dimensions of Vocabulary Development. Studies in Second Language Acquisition, 21(2), 303-317.

KIEFFER, Michael J. \& LESAUX, Nonie K. (2012). Knowledge of Words, Knowledge about Words: Dimensions of Vocabulary in First and Second Language Learners in Sixth Grade. Reading and Writing, 25(2), 347-373.

MARCONI, Diego. (1997). Lexical Competence. Boston, MA : MIT Press. 
LAVOIE, Constance, BÉLANGER, Sophie \& TREMBLAY, Ophélie. (2017). Découvrir les mots et enrichir le langage. Anjou, Québec : Éditions CEC.

LuX-Pogodalla, Veronika \& Polguère, Alain. (2011). Construction of a French Lexical Network: Methodological Issues. Dans Proceedings of the First International Workshop on Lexical Resources, WoLeR 2011. An ESSLLI 2011 Workshop (p. 54-61). Ljubljana. Disponible en ligne sur <https:// hal.archives-ouvertes.fr/hal-00686467>.

MASSERON, Caroline (coord.). (2012). Lexique et écriture. Pratiques, 155-156.

MeArA, Paul M. (1996). The Dimensions of Lexical Competence. Dans G. Grown, K. Malmkajaer \& J. Williams (dir.), Competence and Performance in Language Learning (p. 33-51). Cambridge : Cambridge University Press.

NATION, Paul. (2001). Learning Vocabulary in Another Language. New York : Cambridge University Press.

NisUBIRE, Protais. (2003). Développer la compétence lexicale en classe de français langue seconde. La lettre de l'AIRDF, 33, 20-26.

PAQUETTE, Gilbert. (2002). Modélisation des connaissances et des compétences : un langage graphique pour concevoir et apprendre. Sainte-Foy, Québec: Presses de l'Université du Québec.

POLGUÈRE, Alain. (2016). Lexicologie et sémantique lexicale : notions fondamentales ( $3^{\mathrm{e}}$ éd.). Montréal : Presses de l'Université de Montréal.

POLGUÈRE, Alain. (2014). From Writing Dictionaries to Weaving Lexical Network. International Journal of Lexicography, 27(4), 396-418. <https://doi.org/10.1093/ijl/ecu017>.

POLGUÈRE, Alain \& SIKORA, Dorota. (2013). Modèle lexicographique de croissance du vocabulaire fondé sur un processus aléatoire, mais systématique. Dans C. Ronveaux, C. Masseron \& C. GarciaDebanc (dir.), Enseigner le lexique. Recherches en didactique du français (vol. 5, p. 35-63). Namur : Presses universitaires de Namur.

ScotT, Judith A., SковеL, Bonnie J., \& Wells, Jan. (2008). The Word Conscious Classroom: Building the Vocabulary Reader and Writers Need. New York : Scholastic.

SCHUR, Ellen. (2007). Insights into the Structure of L1 and L2 Vocabulary Networks: Intimations of Small Worlds. Dans H. Daller, J. Milton \& J. Treffers-Daller (dir.), Modelling and Assessing Vocabulary Knowledge (p. 182-203). Cambridge, New York : Cambridge University Press.

SIMARD, Claude. (1994). Pour un enseignement plus systématique du lexique. Québec français, 92, 28-40.

TREMBLAY, Ophélie. (2009). Une ontologie des savoirs lexicologiques pour l'élaboration d'un module de cours en didactique du lexique (Thèse de doctorat, Université de Montréal, Québec). Disponible en ligne sur <https://papyrus.bib.umontreal.ca/xmlui/handle/1866/3598>.

TREmblay, Ophélie. (À paraitre). « Sensibilité lexicale » : l'émergence d'un concept en didactique du lexique. Pratiques.

Tremblay, Ophélie \& AnctiL, Dominic. (2015). Chronique « Au fil des mots ». La conscience lexicale. Vivre le primaire, 28(1), 14-16.

Tremblay, Ophélie, Anctil, Dominic \& Perron, Valérie. (2016, aout). Vers un modèle de la compétence lexicale en didactique du lexique. Communication présentée au symposium « La notion de compétence lexicale en didactique du lexique » dans le cadre du $13^{\mathrm{e}}$ colloque de l'Association internationale pour la recherche en didactique du français (AIRDF), Montréal, Québec. 
TREMBLAY, Ophélie \& GAGNÉ, Andréanne. (2020). Chronique « Au fil des mots ». Aimer les mots pour mieux les apprendre : la sensibilité lexicale au service de l'enrichissement du vocabulaire. Vivre le primaire, 33(2), 16-18.

TREMblay, Ophélie \& Polguère, Alain. (2014). Une ontologie linguistique au service de la didactique du lexique. Actes $d u 4^{e}$ Congrès mondial de linguistique française, 8, 1173-1188. <https:// doi.org/10.1051/shsconf/20140801383>.

TRÉVILLE, Marie-Claude. (2000). Vocabulaire et apprentissage d'une langue seconde : recherches et théories. Outremont, Québec : Éditions Logiques.

WILKS, Clarisssa \& MEARA, Paul. (2002). Untangling Word Webs: Graph Theory and the Notion of Density in Second Language Word Association Networks. Second Language Research, 18(4), 303-324.

ZareVA, Alla, Schwanenflugel, Paula \& Nikolova, Yordanka. (2005). Relationship between Lexical Competence and Language Proficiency: Variable Sensitivity. Studies in Second Language Acquisition, 27(4), 567-595. <https://doi.org/10.1017/S0272263105050254>.

\section{NOTES}

1. Calaque \& David, 2004 ; Garcia-Debanc, Masseron \& Ronveaux, 2013 ; Grossmann, Paveau \& Petit, 2005 ; Grossmann \& Plane, 2008.

2. Par exemple: La Lettre de l'AIRDF, 64, "La didactique du lexique» (2018); Pratiques, 155-156, "Lexique et écriture» (2012); Les Langues Modernes (revue de l'Association des professeurs de langues vivantes), 103(1), « Enseigner le lexique » (2009).

3. Deux symposiums à l'AIRDF $(2016,2019)$, trois colloques à l'ACFAS $(2015,2017,2019)$ et une journée d'étude «Enseigner le lexique» à la Haute École pédagogique de Fribourg (Suisse) en 2015.

4. Voir <https://crisco2.unicaen.fr/des/>.

5. Voir <www.cnrtl.fr>.

6. Voir <www.lextutor.ca/conc/fr/>.

7. Voir <www.manulex.org/fr/manulex/request.html>.

8. Voir <www.franqus.ca/MELS/liste_orthographique/outil_de_recherche/>.

\section{AUTEURS}

\section{OPHÉLIE TREMBLAY}

Université du Québec à Montréal

\section{DOMINIC ANCTIL}

Université de Montréal 